\title{
Could we have done better with COVID-19 in nursing homes?
}

\author{
Katarzyna Szczerbińska ${ }^{1}$
}

Published online: 16 July 2020

(c) European Geriatric Medicine Society 2020

According to a communication published on June 4, 2020 by The European Centre for Disease Prevention and Control (ECDC), 31-66\% of all deaths, depending on country, due to COVID-19 were among residents in long-term care facilities (LTCF) [1]. The COVID-19 pandemic has uncovered many challenges that have probably existed in LTCF for years, yet few people would have thought they could lead to such dramatic effects. One may blame the new coronavirus for the tragic outcomes, but the bottom line is that LTCFs were simply not prepared for the outbreak, and they were "abandoned" at the time when all health care settings struggled with shortage of personal protective equipment (PPE) and testing [2]. The epidemic is not over yet. Moreover, during the potential next wave, we may once again witness a disaster, if we do not back-reflect on the events of the past months and learn a lesson on how to avoid such a scenario. Therefore, right now is the time to analyse which features of LTC did and did not work, and specify which measures may be undertaken in the nearest weeks, and in the future.

\section{Organization and structure limitations}

By design, LTC institutions are neither equipped with specialist medical gear, nor staffed with specialized medical staff. Their role is to reduce the costs of treatment by relieving hospitals from care for chronically ill patients and shortening the length of their stay in expensive acute care wards. Housing conditions in these facilities depend on their economic condition and differ between the countries. For example, LTCFs in Belgium provide fairly spacious rooms (typically over $16 \mathrm{~m}^{2}$ per person), while only 6 square meters per person in the room are required in residential homes in Poland. The rooms in newly designed home accommodate

Katarzyna Szczerbińska

katarzyna.szczerbinska@uj.edu.pl

1 Laboratory for Research on Aging Society, The Chair of Epidemiology and Preventive Medicine, Medical Faculty, Jagiellonian University Medical College, Kraków, Poland
1-2 persons, but there are still many facilities situated in old buildings which have rooms for 3 and more residents providing them only a small personal space. They do not have an isolation room for an infected resident. Also, quarantine or cohorting is not easy to organize there. Most of LTCFs contain around 100 beds. While smaller facilities are economically disfavored, they provide a more family-like environment and a better quality of care [3], and may be safer in terms of limiting spreading of infections to a large number of residents and staff. The study of 9395 nursing homes in the US showed that the probability of having a COVID-19 case was higher for larger facilities at urban locations [4].

The way LTC facility buildings are designed and renovated should be rethought in the future so as to divide them into smaller wards with additional separate rooms for residents who need isolation due to infection, and to provide the possibility for family members to stay with the resident at the time of her/his death. This concerns not only SARS$\mathrm{CoV} 2$, but may reduce spreading of other infections as well, such as influenza, Hemophilus influenzae, Pneumococcus pneumoniae, Norovirus or Clostridium difficile, and in turn save lives and reduce costs of treatment.

\section{Lack of infection control}

The Centers for Disease Control and Prevention (CDC) developed recommendations to keep COVID-19 from entering LTC facilities: everyday active screening residents for early detection, preventing spread of infection in the facility, and assuring optimized personal protective equipment (PPE) supply. The residents with suspected or confirmed COVID-19 should be separated and monitored 3 times daily for temperature and oxygen saturation to identify those who require transfer to hospital [5]. Additionally, the European centre, ECDC, suggested implementing a daily surveillance routine to monitor the residents for typical symptoms (cough, fever, sore throat, shortness of breath, sudden onset of anosmia, ageusia or dysgeusia) and atypical symptoms (headache, chills, muscle pain, fatigue, vomiting, diarrhea), 
as well as signs (oxygen saturation via pulse oximetry less than $95 \%$, respiratory rate over $25 / \mathrm{min}$ ). The resident or staff with suspected COVID-19 should be tested promptly, and when confirmed, all residents and staff in the facility should undergo weekly or biweekly tests, if available [1]. ECDC developed a registration form to collect data on all residents with positive PCR test or symptoms (cough, temperature over $38^{\circ} \mathrm{C}$, dyspnea) and to keep further track of each case, including symptoms, hospitalization, death and recovery. However, these measures do not prevent infection, but rather focus on the detection of the first case in the facility and monitoring the following situation. At the time when the first case is found, one may be sure that $60-70 \%$ of residents are already infected, half of them are asymptomatic [6] and around one-fourth will die [7, 8]. Therefore, more effective procedures are needed to protect the residents from being exposed to the infection being brought into the institution by others: for example, regular testing of staff in advance, and a labour law assuring compensation when the worker is ill to avoid a person with symptoms coming to work. Regular staff training is another important way to raise awareness on how to prevent the spread of an infection. For example, the measures introduced in Hong Kong during the SARS epidemy in 2003 have since then remained in force, and recently, they enabled an immediate reaction to the threat of COVID-19. All health care institutions kept PPE in stock (exchanged every 3 months). Policies learned from SARS, such as social distancing, rigorous hygiene practice, or using masks, were swiftly adopted by the health care staff and all citizens [9]. That was not the case in Europe, where many LTC facilities were poorly equipped, and staff was not trained on how to protect themselves and the residents.

LTC facilities are organized differently, depending on country, within the frame of social or health care sector. Nevertheless, they should adopt infection control rules existing in hospitals, because they provide care for vulnerable older, chronically ill persons, who are at higher risk of complications and death due to any infection.

\section{Education via e-learning}

In most countries, there are established structures for control of hospital infections: infection control teams, epidemiological nurses, obligatory staff training on prevention of nosocomial infections. No such trainings are available for the staff in most LTC facilities, mainly due to their high cost and lack of regulations that would call for obligatory trainings. Hence, upon the breakout of COVID-19, the basic rules of protection had to be learnt immediately and adopted universally. How to wash hands and use PPE? And how to behave in a safe way? Similarly, the pandemic triggered a universal motivation to adopt e-learning, which has earlier been a marginal approach.

Therefore, obligatory trainings (for example, via Internet) for new nursing home staff, repeated periodically to revise the rules and best practices, might improve the staff's confidence in protecting the residents and themselves from COVID-19 and other infections.

\section{Insufficient human resources}

In most residential homes or care homes, medical care is provided by a general practitioner or a family doctor, whereas in most nursing homes, physicians are employed on-site. However, no specific requirements need to be fulfilled in terms of medical specialization. Therefore, physicians employed at LTC facilities are not necessarily geriatricians. The unique example is a specialization of "elderly care physician" in the Netherlands [10]. The Special Interest Group in Long-Term Care of the European Geriatric Medicine Society (SIG-LTC of EUGMS) advocates for implementing a minimum standard of care in the nursing homes, which includes an obligatory training in LTC for physicians, which might improve the quality of care for residents and their protection from infections like SARS-CoV-2 [11]. On the other hand, this requirement should not become an additional barrier discouraging physicians from working in LTC facilities. The implementation of new technologies, such as "telegeriatrics", could improve the accessibility of geriatric and other specialist consultations for residents in LTC facilities, which would certainly help physicians and other professionals working there to provide better care.

Ageing of the society and the increasing number of chronically ill persons are the challenges to the health care systems, due to insufficient human resources and relatively high staff turnover. The shortage of skilled professionals working in LTC concerns not only physicians, but also nurses and care assistants [12]. Therefore, in the last two decades, the registered nurses have been gradually replaced by care assistants in many LTC facilities all over the world. However, work in LTC is highly demanding in terms of both physical and psychological burden. Confronted with a relatively low financial compensation, many LTC staff decide to leave the profession after some time and search for easier job. Therefore, the main managerial issue is how to attract and keep the care providers at LTCFs.

One could clearly appreciate during the COVID-19 pandemic that how much the qualified staff matters. The cross-sectional analysis of 215 nursing homes in Connecticut showed that every $20 \mathrm{~min}$ (per resident day) increase in registered nurse (RN) staffing was associated with a $22 \%$ reduction in the number of confirmed COVID-19 cases (in the facilities with at least one confirmed case) and $26 \%$ fewer 
COVID-19 deaths in the facilities with at least one death due to this infection [13].

During this difficult time, it occurred that many of the LTC staff had other part-time jobs elsewhere (at hospitals, outpatient clinics or other nursing homes), which increased the risk of transmitting the infection between the facilities. This situation is caused mainly by the very low salaries in LTC - the lowest in healthcare sector-making LTCFs an unattractive workplace, facing serious difficulties in keeping the skilled staff in the institution for longer. Moreover, the pandemic has forced a complete reorganisation of the work, introducing multi-day shifts disregarding the workers' rights. In some countries, additional financial compensation and special health insurance for staff who stayed to care for residents were assured. Many staff members have accepted these conditions with understanding, despite the increased risk of infection and isolation. However, there were also some who refused to continue working.

The epidemic revealed an urgent need for a serious discussion at the national and EU level towards elaborating a strategy for the development of the LTC sector. Such strategy should assure maintenance and sustainability of LTCFs through strengthening the LTC staff. It is crucial to increase the prestige of professions performed in LTC. This can be accomplished with the use of different incentives, not only financial, but also, for example, by offering professional career development paths, updating qualifications, opportunities for education and certification of the new skills.

\section{Emotional burden of the staff}

The pandemic revealed unexpected emotions arise at LTCFs, such as fear, stress, and even panic among residents and workers. These emotions may be regarded as unprofessional, yet they constitute a very human reaction to the unknown and unpredictable. Frontline health workers involved in the direct care for patients with COVID-19 had a higher risk of symptoms of depression, anxiety, insomnia, and distress [14]. There were also facilities where employees did not show up for work because of the fear for their health and the health of their loved ones. The main reason for that reaction was the lack of knowledge on how to behave and protect oneself and others, as well as the lack of procedures, which have been later developed on an ad hoc basis.

Therefore, appropriate procedures and staff trainings should be introduced (as it was in South Korea and Hong Kong) to make the staff confident that they know what and when to do properly. A potential success of such measures may strengthen the LTC workers, making them proud that they managed, and increase their future dedication to their job.

\section{Ethical crisis}

The SARS-CoV-2 pandemic has raised many ethical issues. Suspension of visits by relatives and isolation of residents were not entirely new measures, as they were already practised during previous influenza epidemics, but the recent ban has been substantially extended and strictly enforced. However, modern technologies, such as laptops, tablets and smart phones, provide the option to see each other while talking, which helps maintaining frequent contact with the patient's family and friends. Hence, facilities should make these measures of communication more accessible to the patients.

The most painful aspect of the pandemic concerned the infected residents dying without the possibility to say goodbye to their relatives, and the extraordinary restrictions concerning the burial ceremony. In some facilities, the infected resident may have been placed in a separate room, and special precautions have been taken to allow families to be present when the loved one passes away. Even though this possibility was not offered frequently, one should not think that the residents were dying alone. Probably their situation was better in nursing homes than that of hospital patients. In many cases, family members do not visit the residents very often, and it is the nursing home staff who becomes the very close everyday caregiver of the residents. Therefore, the numerous deaths of the residents might have had a detrimental impact on the mental health of care assistants and nurses, causing an immense psychological burden that usually did not receive enough attention. Hence, facilities should develop programs supporting both the relatives and the nursing home staff following the death of a resident, allowing reflection and relief of emotions to reduce the burden.

The review of the recently published COVID-19 pandemic care recommendations showed that most of the guidelines focused on SARS-CoV-2 infection prevention and control, giving much less attention to palliative care for the residents with COVID-19. They were limited to organization of the end-of-life visits, advanced care planning (ACP) documentation, and clinical decision-making concerning treatment preferences at the end of life. The other important aspects of palliative care were rarely addressed, for example, symptoms management at the end of life, psychological, social and spiritual needs of both the resident and their family, care for patients with dementia, and supporting the staff and the family in bereavement. These aspects should be granted greater attention in future guidelines [15].

Although ACP had been extensively recommended already before the pandemic, its implementation differs a lot between countries depending on the local cultural 
and legal context [16]. During the unexpected and quickly evolving threat of SARS-CoV-2, ACP suddenly became a very desirable tool in making medical decisions. However, in many COVID-19 pandemic recommendations, the concept of ACP has been narrowed down to advanced directives (expressed as "do not hospitalize" or "do not resuscitate"). In some countries, the guidelines literally advise to avoid admission to hospital (in Belgium, Scotland), while in others (like the US), they make the decision on the hospitalization dependent on the clinical condition [15]. As a result, less than $1 \%$ of nursing home residents in the Netherlands died of COVID-19 in a hospital, while $30 \%$ of them remained in the facilities to die.

So, is ACP a proper solution? Probably yes, but under certain conditions. First, ACP should be treated much broader than just the " 5 wishes" discussion. Second, it should be started in advance—not in "the last minute"-with the resident capable to make decisions. Finally, ACP should be conducted by a staff member of the nursing home having a good supportive relationship with the patient [17]. Importantly, there is a fine line between honest ACP and its misuse for a negative triage. Even when a patient makes a wish not to be hospitalized or resuscitated, the final evaluation of the indications should be made by a physician to avoid unjustified disqualifying of the resident from treatment. Lack of procedures always results in conflicting decisions. Therefore, there is an urgent need to develop recommendations for dealing with such extreme situations. Policies and guidelines would give the staff the confidence to act and a sense of security, necessary for reducing the stress associated with handling extreme scenarios.

While struggling for lives, we are still obliged to assure the quality of dying for those who have no chance to survive. Palliative care plans should be implemented for those patients immediately in every setting. The PACE project conducted in $6 \mathrm{EU}$ countries showed that despite most nurses and care givers were relatively knowledgeable about the general principles of care for nursing home residents, in some countries, their knowledge of end-of-life care was rather poor [18]. Therefore, mandatory trainings on end-oflife care should be promoted among all LTC staff.

And finally, we have witnessed diverse reception of the staff's performance by the public, ranging from calling them heroes to openly expressing hatred, with examples of vandalism and refusal of support. Such 'trampling on others' in panic, rarely seen in the time of peace, is triggered by a primary fear for their lives and those of their loved ones. The only way to limit such hateful behavior relies on national governments and EC convincing the society that they control the situation and have a strategy. It seems that in this pandemic country governments did better than EC and EU institutions, although none of them managed to completely prevent casualties.

\section{Lack of plan for a crisis situation}

Many issues related to protection from COVID-19 in LTC facilities have been identified [19]. Referring to the operational framework for a coordinated response to COVID-19 developed by Behrens and Naylor, we find that many nursing homes experienced a crisis or a catastrophic condition [20]. Therefore, we must put forward an action plan for the future!

In 2019, there were five workshops dedicated to LTC held by the EC Directorate-General for Employment, Social Affairs and Inclusion. They aimed to point out the issues to be involved in an action plan of the new EC. The panel of invited experts identified a range of challenges and proposed potential solutions, summarized as a list of the recommendations to the EC [21]. Many of the issues related to the COVID-19 pandemic described in this Editorial were actually addressed by these recommendations, including improvement of LTC infrastructure and organisation, development of minimum qualification standards (as in the health care sector) and life-long learning and career paths in LTC. Thus, an extensive diagnosis is already on the table of decision makers, along with recommendations and guidelines. Now is the time for the EC to take appropriate actions and develop a strategy for LTC to effectively protect the lives of their residents.

\section{Compliance with ethical Standards}

Conflict of interest None declared.

Ethical approval Not reqiured.

Informed consent The author gave consent for publication.

\section{References}

1. Public Health Emergency Team ECDC, Danis K, Fonteneau L, Georges S, Daniau C, Bernard-Stoecklin S, Domegan L et al (2020) High impact of COVID-19 in long-term care facilities, suggestion for monitoring in the EU/EEA, May 2020. Euro Surveill. https://doi.org/10.2807/1560-7917.ES.2020.25.22.2000956

2. Abbasi J (2020) "Abandoned" nursing homes continue to face critical supply and staff shortages as COVID-19 toll has mounted. JAMA. https://doi.org/10.1001/jama.2020.10419

3. Baldwin R, Chenoweth L, Rama MD, Wang AY (2017) Does size matter in aged care facilities? A literature review of the relationship between the number of facility beds and quality. Health Care Manage Rev 42(4):315-327. https://doi.org/10.1097/HMR.00000 00000000116

4. Abrams HR, Loomer L, Gandhi A, Grabowski DC (2020) Characteristics of US nursing homes with COVID-19 cases. J Am Geriatr Soc. https://doi.org/10.1111/jgs.16661

5. Centres for Disease Control and Prevention (2020) Key strategies to prepare for COVID-19 in long-term care facilities (LTCFs) 
(page issued in April 15, 2020) https://www.cdc.gov/coronaviru s/2019-ncov/hcp/long-term-care-strategies.html. Accessed on 18 Jun 2020

6. Kimball A, Hatfield KM, Arons M, James A, Taylor J, Spicer $\mathrm{K}$ et al (2020) CDC COVID-19 Investigation Team. Asymptomatic and presymptomatic SARS-CoV-2 infections in residents of a long-term care skilled nursing facility-King County, Washington, March 2020. Morbidity Mortality Weekly Rep 69(13):377-381

7. Arons MM, Hatfield KM, Reddy SC, Kimball A, James A, Jacobs JR et al (2020) Presymptomatic SARS-CoV-2 infections and transmission in a skilled nursing facility. N Engl J Med 382(22):2081-2090. https://doi.org/10.1056/NEJMoa2008457

8. Patel MC, Chaisson LH, Borgetti S, Burdsall D, Chugh RK, Hoff CR et al (2020) Asymptomatic SARS-CoV-2 infection and COVID-19 mortality during an outbreak investigation in a skilled nursing facility. Clin Infect Dis. https://doi.org/10.1093/cid/ciaa7 63

9. Lum T, Shi C, Wong G, Wong K (2020) COVID-19 and long-term care policy for older people in Hong Kong. J Aging Social Policy. https://doi.org/10.1080/08959420.2020.1773192

10. Koopmans RT, Pellegrom M, van der Geer ER (2017) The Dutch move beyond the concept of nursing home physician specialists. J Am Med Dir Assoc 18:746-749

11. O'Neill D, Briggs R, Holmerova I, Samuelsson O, Gordon AL, Martin FC, on behalf of the Special Interest Group in Long Term Care of the European Geriatric Medicine Society (2020) COVID19 highlights the need for universal adoption of Standards of Medical Care for Physicians in Nursing Homes in Europe. Eur Geriatr Med. https://doi.org/10.1007/s41999-020-00347-6

12. Michel JP, Ecarnot F (2020) The shortage of skilled workers in Europe: its impact on geriatric medicine. Eur Geriatr Med 11:345-347. https://doi.org/10.1007/s41999-020-00323-0

13. Li Y, Temkin-Greener H, Gao S, Cai X (2020) COVID-19 infections and deaths among connecticut nursing home residents: facility correlates. J Am Geriatr Soc. https://doi.org/10.1111/jgs.16689

14. Lai J, Ma S, Wang Y, Cai Z, Hu J, Wei N et al (2020) Factors associated with mental health outcomes among health care workers exposed to coronavirus disease 2019. JAMA Network
Open 3(3):e203976. https://doi.org/10.1001/jamanetworkopen 2020.3976

15. Glissen J, Pivodic L, Unroe KT, Van den Block L (2020) Van den Block (2020) International COVID-19 palliative care guidance for nursing homes leaves key themes unaddressed. J Pain Symptom Manage. https://doi.org/10.1016/j.jpainsymman.2020.04.151

16. Andreasen P, Finne-Soveri H, Deliens L, Van den Block L, Payne S, Gambassi G et al (2019) Prevalence of written advance directives in European long-term care facilities: a cross-sectional survey. BMJ Support Palliat Care 21:1-10. https://doi.org/10.1136/ bmjspcare-2018-001743

17. Phenwan T, Sixsmith J, McSwiggan L, Buchanan D (2020) A narrative review of facilitating and inhibiting factors in advance care planning initiation in people with dementia. Eur Geriatr Med 11:353-368. https://doi.org/10.1007/s41999-020-00314-1

18. Smets T, Pivodic L, Piers R, Pasman HRW, Engels Y, Szczerbińska K et al (2018) The palliative care knowledge of nursing home staff. The EU FP7 PACE cross-sectional survey in 322 nursing homes in six European countries. Palliat Med 32(9):1487-1497. https://doi.org/10.1177/0269216318785295

19. Bakerjian D (2020) Coronavirus Disease 2019 (COVID-19) and Safety of Older Adults (page issued in April 21, 2020) https:// psnet.ahrq.gov/primer/coronavirus-disease-2019-covid-19-andsafety-older-adults. Accessed 18 June 2020

20. Behrens LL, Naylor MD (2020) "We are Alone in This Battle": a framework for a coordinated response to COVID-19 in nursing homes. J Aging Soc Policy 32(4-5):316-322. https://doi. org/10.1080/08959420.2020.1773190

21. Mutual Learning Workshops on Long-term Care. Final Outcome Report (2019) EUROPEAN COMMISSION, Directorate-General for Employment, Social Affairs and Inclusion, Brussels, July 2019.

Publisher's Note Springer Nature remains neutral with regard to jurisdictional claims in published maps and institutional affiliations. 